\title{
HYDROGELS THE THREE DIMENSIONAL NETWORKS: A REVIEW
}

\author{
TRINADHA RAO M., PHANINDRA CHVS*, YAMINI M., PRASAD C. H. \\ Vignan Institute of Pharmaceutical Technology, Duvvada, Visakhapatnam \\ ${ }^{*}$ Email: thrinadh_81@rediffmail.com
}

Received: 15 Sep 2020, Revised and Accepted: 17 Nov 2020

\begin{abstract}
Hydrogel products constitute polymeric materials as a group, the hydrophilic structure which renders them the capability of holding large amounts of water in their 3-dimensional networks. Extensive employment of those products during a number of commercial and environmental areas of application is taken into account to be of prime importance. Needless to say, natural hydrogels were gradually replaced by synthetic types thanks to their higher water absorption capacity, long service life, and wide sorts of raw chemical resources. Literature on this subject was found to be expanding, especially within the scientific areas of research. However, variety of technical reports publications and handling hydrogel products from the points of view of engineering were examined to overview technological aspects covering this growing the field of research. the first objective of this text is to review the literature concerning classification of hydrogels on different bases, chemical characteristics and physical characteristics of those products, and technical feasibility of their best utilization. It also involved the technologies adopted for hydrogel production along-side process and design implications, optimized conditions, and the block diagrams of the preparation process. Recent forms of hydrogel material generations are also presented in some details that are innovative in nature.
\end{abstract}

Keywords: Hydrogel, 3-Dimentional network, Polymers

(C) 2021 The Authors. Published by Innovare Academic Sciences Pvt Ltd. This is an open access article under the CC BY license (https://creativecommons.org/licenses/by/4.0/) DOI: https://dx.doi.org/10.22159/ijcpr.2021v13i1.40823. Journal homepage: https://innovareacademics.in/journals/index.php/ijcpr

\section{INTRODUCTION}

Hydrogels are defined as the 3-dimensional network of hydrophilic cross-linked polymer which don't dissolve in water but can swell in water or can respond to the changes occurring the environmental stimuli; they can form polymeric networks; hence they are hydrophilic in nature because they can absorb a great amount of water. Hydrogels are durable chemically and can easily disintegrate or dissolve [1]. Hydrogels are hydrophilic in nature hence these are also called as hydrophilic gels they form a network of polymer chains sometimes can be found as colloidal gels containing water as dispersion medium [2].

The linkage between the hydrogels was formed by molecular entanglements and by the secondary forces like hydrogen bonding, ionic bonding, or hydrophobic forces, hence are called as physical or reversible gels. The physical gels are easily dissolved by altering in environmental conditions such as temperature, $\mathrm{pH}$, ionic strength of solution [3]. The gels may be non-charged or charged based on the functional groups existing in the structure. The charged hydrogels show changes in shape when an electrical field is introduced. Hydrogels can be manufactured from any water-soluble polymer with wide range of physical properties and chemical compositions in bulk [4].

A variety of physical forms such as microparticles, nanoparticles, slabs, coatings, or films can be formulated by using hydrogels. Hydrogels are also used everywhere universally in clinical practices and investigational medicine for a variety of applications like regenerative medicine and tissue engineering, cellular immobilization, diagnostics, separation of biomolecules and cells. In comparison with other synthetic biomaterials, hydrogels resemble to be the living tissues closely in their physical properties due to their relatively high water content and soft rubbery consistency [5]. Hydrogels are also called as intelligent gels or smart gels. Hydrogels can perceive stimuli and respond by exhibiting the changes in their chemical or physical behaviour [6].

\section{Properties of hydrogels}

Physical and chemical properties

In spite of a great deal of progress, a fundamental knowledge of gel homes isn't but enough for a rational layout of novel gel systems. For such designs, it's far crucial to realize how solute molecules have interaction with the gel, particularly how they partition among the gel segment and the encircling liquid segment. Partitioning depends on most important consequences: length exclusion and molecular enchantment/repulsion $[7,8]$.

Hydrogels are cross-linked polymer networks swollen during a liquid medium. The imbibed liquid is a selective filter to permit the free diffusion of some solute molecules, while the polymer network is a matrix to carry the liquid together. Hydrogels are absorbed from $10-20 \%$ (arbitrary lower limit) Up to thousands of times of their dry weight in water. The character of the water during a hydrogel can determine the total permeation of nutrients into and cellular products out of the gel. When any dry hydrogel begins to soak up water, the primary water molecules entering the matrix will hydrate the foremost polar, hydrophilic groups, results in the primary bound water. Because of hydrated polar groups, the network swells and exposes hydrophobic groups, which will also interact with water molecules that are resulting in the formation of hydrophobicallybound water, or secondary bound water [9].

Primary and secondary bound water are combined often and easily called the entire bound water. After the polar and hydrophobic sites have interacted with and bound water molecules, the network will imbibe additional water to the osmotic drive of the network chains towards infinite dilution. This extra swelling is opposed by the covalent or physical crosslink, resulting in an elastic network retraction force [10]. Thus, the hydrogel reaches an equilibrium swelling level. the extra swelling water that's imbibed after the ionic, polar and hydrophobic groups saturated with bound water is called as bulk water or free water and is assumed to fill the space between network chains and or the middle of larger pores, macropores or voids. Because the network swells, if the network chains or crosslink are degradable, the gel will begin to disintegrate and dissolve, at a rate counting on its composition. There is the variety of methods employed by researchers to estimate the relative amounts of free and bound water as fractions of the total water content. All of them are controversial since there's proton NMR evidence that the interchange of water molecules between the bound and free states are very rapid, perhaps as fast together with water $\left(\mathrm{H}_{2} \mathrm{O}\right)$ molecule every 10-9s [11]. The three major methods won't to characterize water in hydrogels are supported the use of small molecular probes, 
NMR and DSC When probe molecules are used, the labelled probe solution is equilibrated with the hydrogel, and the concentration of the probe molecule in the gel at equilibrium is then measured. Assuming that only the free water within the gel can dissolve the probe solute, one can calculate the free water content from the quantity of the imbibed probe molecule and therefore the known (measured) probe molecule concentration within the external solution. Then the bound water is obtained by difference of the measured total water content of the hydrogel and calculated free water content [12].

The use of DSC is predicated on the idea that only the free water could also be frozen, so it's assumed that the endotherm measured when warming the frozen gel represents the melting of the free water, which value will yield the quantity of free water in the HG sample being tested. The bound water is obtained by the difference of the measured total specimen and the calculated free water content. In another formulation, swelling is that the property to soak up water and retain it for a relative while. It are often evaluated by measuring the dry weight and therefore the swollen-state weight and computing either a ponderal variation (water uptake) or a volume of adsorbed solvent (both the quantities are considered as percentages) [13].

The evaluation of swelling is that the principal assay to be performed on hydrogel samples, because it are often a measure for several of their properties: cross linking degree, mechanical properties, degradation rate then on. For several gels, the evaluation of swelling and swollen state stability is that the simplest, cheapest and surest thanks to discriminate between cross-linked gels and, therefore the not cross-linked original polymer [14].

\section{Mechanical properties}

The mechanical properties can vary and be tuned depending on the purpose of the fabric. it's possible to get a gel with higher stiffness increasing the cross-linking degree or lowering it by heating the fabric. The changes in mechanical properties link to a good range of variables and causes and different analysis must be made consistent with the fabric, the conditions and therefore the aim of the study. Forinstance, while gelatin show a clear increase in Young Modulus through crosslinking silk fibroin features a very high Young Modulus, but after the revitalization it'll decrease. These properties are often evaluated by a Dynamic Mechanical Analysis (DMA) device or a rheometer, consistent with the thousands of techniques available on the market, which will be no further discussed here. It's important to notice that during a hydrogel, the Young Modulus is that the results of the union between water and gel matrix. If we've to seeds osteoblast cells we'll need a more stiff material than if we culture adipocyte, an equivalent rationale is valid for the event of a heterogeneous prosthesis, for instance, substitute for the intervertebral disk [15].

\section{Porosity and permeation}

Pores can be also formed in hydrogels by the phase separation during the synthesis, or they will exist as smaller network pores within the network. The typical pore size, the pore size distribution, and therefore the pore interconnections are important factors of a hydrogel matrix they are often difficult to quantify and are usually included together within the parameter called tortuosity. The effective diffusion path length across a HG film barrier is estimated by the film thickness the ratio of the pore volume fraction divided by the tortuosity. These factors are most affected by the composition and crosslink density of the hydrogel polymer network [16].

Labelled molecular probes of a variety of molecular weights (MWs) or molecular sizes are wont to probe pore sizes in hydrogels. Poresize distributions of hydrogels strongly suffer from three major factors: Concentration of the chemical cross-links of the polymer strands. That concentration is decided by the initial ratio of crosslinker to monomer. Concentration of the physical entanglements of the polymer strands. That concentration is decided by the initial concentration of all polymerizable monomers within the solution. Net charge of the polyelectrolyte hydrogel.

That charge is decided by the initial concentration of the cationic and/or anionic monomer. These three factors are often quantified using the composition of the hydrogel, by the nominal concentrations of monomer and the cross-linker. The porous structure of a hydrogel is additionally suffering from the properties of the encompassing solution, especially by dissolved ionic solutes (Donnan effects) and by dissolved uncharged solutes which partition unevenly between the gel phase and therefore the solution phase (Osmotic effects).

For rational design of hydrogels, it is useful to understand that the pore-size distribution depends up on the hydrogel characterization which is expressed usually by $\% \mathrm{C}$ and a light. Most techniques wont to investigate the porosity of hydrogels are limited because they require the temperature to get al. tered, causing the gel to shrink, swell or require mathematical manipulation and assumption, which may introduce unwanted artifacts. Porosity may be a morphological feature of a cloth, which will be simply described because the presence of void cavity inside the majority. It is useful to regulate the porosity in many devices for a good variety of applications, like optimal cell migration in hydrogel-based scaffolds or tuneable lode/release of macromolecules [17].

In a sample, pores can show different morphologies: they will be closed, open as a blind end or interconnected, again divided in cavities and throats. These porosities can had been studied and evaluated in papers within the past decades employing a various spectrum of techniques. First of all, porosity are often evaluated by theoretical methods, like unit cube analysis, mass technique. Archimedes method, liquid displacement method. These analysis are commonly including optical and electronic microscopy [18]. Other interesting techniques are the mercury porosimetry, which is quanti based on Washburn's equation, the inconvenience of being a destructive assay, the gas pycnometry, the gas adsorption (that can be issued using different procedures like small quantity adsorption, monolayer and multilayer adsorption), liquid extrusion porosity, an assay that allows to evaluated sample's permeability too, capillary flow porosity, again a test supported Washburn's equation. Furthermore another important assay is the Micro-CT, which is also called X-ray Microtomography, a relative new imaging technique, simply described as a non-destructive high resolution radiography, capable of qualitative and quantitative assays on samples and the evaluation of their pore interconnections. Between the quantitative assays which will be performed, micro-CT can give information on the average pore size, pore size distribution, pore interconnection, struts/walls thickness and anisotropy/isotropy of the sample (in the sense of presence/absence of preferential orientation of the pores). It is yet, nowadays, an expensive technique both in term of cash and time. Microscopy techniques are often utilized in thousands of various assays involving hydrogels. They both are involved in the qualitative and quantitative tests, from simple morphological assessing of material's properties to more complex biocompatibility assays. Briefly, by microscopy techniques topography and surface morphology are often assessed. These techniques are often divided in many classes, by increasing magnification power: optical microscopy (OM), stereo microscopy (SM), microscopy (SEM and TEM), tunnelling microscopy (STM), atomic force microscopy (AFM) $[19,20]$.

\section{Crosslinking}

Crosslinking isn't properly a property of hydrogels, while it's more of aexplanation for all the opposite properties of the fabric itself. The crosslinking degree are often correlated to basically every characteristic of a hydrogel. the character of the crosslinking can vary a lot. Indeed, the hydrogel's networks are often obtained in many different ways. The processes are divided into two big categories: first one is called physical crosslinking that happens, for example because of hydrophobic interactions between chains, ionic interactions between a polyanionand a (complex coacervation) polycation or ionic interactions between a (ionotropic hydrogel) polyanion and multivalent cations [20].

The second category comprises the chemical bound gels. The crosslinking can be occured by ultraviolet (UV) irradiation, heating or chemical crosslinking via crosslinker with an enormous ensemble of reactions, like Michael'sreaction, Michaelis-Arbuzov reaction, nucleophile addition then on. By controlling the degree of crosslinking is feasible to tune the property of the fabric and 
optimize it for several different applications getting theoretically, during this way, a good spectrum of applications ranging from an equivalent original polymer [21].

\section{Classification}

\section{Classification of hydrogels}

Hydrogels are often classified into two groups counting on the character of the cross-linking reaction (table no 1,2 and 3 ). If the cross-linking reaction involves the formation of covalent bonds, then the hydrogels are termed as permanent hydrogel. If the hydrogels are formed by the physical interactions, viz. molecular entanglement, ionic interaction and hydrogen bonding, among the polymeric chains then the hydrogels are termed as physical hydrogels [22]. Hydrogels also can be categorized as conventional and stimuli-responsive hydrogels. Conventional hydrogels are the cross-linked polymer chains which absorb water when put in an aqueous media and there's no change within the equilibrium swelling with the change within the $\mathrm{pH}$, temperature or field of the encompassing environment while the stimuli-responsive hydrogels are the polymeric networks which change their equilibrium swelling with the change of the encompassing environment [23].

Table 1: Depending on cross-linking nature of hydrogels

\begin{tabular}{ll}
\hline S. No. & Type of hydrogel \\
\hline 1 & Permanent hydrogels \\
2 & Physical hydrogels \\
3 & Conventional or Stimuli-responsive hydrogels \\
\hline
\end{tabular}

Table 2: Depending upon method of preparation

\begin{tabular}{ll}
\hline S. No. & Type of hydrogels \\
\hline 1 & Homopolymer hydrogels \\
2 & Copolymer hydrogels \\
3 & Multipolymer hydrogels \\
4 & Interpenetration polymeric hydrogels \\
\hline
\end{tabular}

Table 3: Other types of hydrogels

\begin{tabular}{ll}
\hline S. No. & Intelligent (or) smart hydrogels \\
\hline 1 & PH sensitive hydrogels \\
2 & Temperature-sensitive hydrogels \\
3 & Complexing hydrogels \\
4 & Insitu hydrogels \\
\hline
\end{tabular}

\section{Intelligent (or) smart hydrogels}

Hydrogels may exhibit swelling behaviour hooked in to the external environment. Over the past $30 \mathrm{y}$ there has been a big interest within the development and analysis of environmentally or physiologically responsive hydrogels. Due to the changes in their external pH, temperature, ionic strength, nature and composition of the swelling agent, enzymatic or reaction, and Electrical or magnetic stimuli, environmentally responsive materials show drastic changes in their swelling ratio [24]. In most responsive networks, a juncture exists at which this transition. a stimulating characteristic of various responsive gels is that the mechanism causing the network structural changes are often entirely reversible in nature. the power of $\mathrm{pH}$-or temperature-responsive gels to exhibit rapid changes in their swelling behavior and pore structure in response to changes in environmental conditions lend these materials favorable characteristics as carriers for bioactive agents, including peptides and proteins. This sort of behavior may allow these materials to function in self-regulated, pulsatile drug delivery systems [25].

\section{pH sensitive hydrogels}

One among the foremost widely studied sorts of physiologically responsive hydrogels is pH-responsive hydrogels. $\mathrm{pH}$ sensitive hydrogels are swollen ionic networks that contain either acidic or basic pendant groups. In aqueous media of appropriate $\mathrm{pH}$ and ionic strength, the pendant groups can ionize, developing fixed charges on the gel. All the ionic materials show $\mathrm{pH}$ and ionic strength sensitivity. The swelling forces developed in these systems are increased over those of non-ionic materials. This increase in swelling force is thanks to the localization of fixed charges on the pendant groups. As a result, the mesh size of the polymeric networks can change significantly with small $\mathrm{pH}$ changes.

Examples: poly (acryl amide), poly (acrylic acid), poly (methacrylic acid) [26].

\section{Temperature-sensitive hydrogels (or) thermo gels}

Another class of environmentally sensitive gels exhibits temperature-sensitive swelling behavior thanks to a change within the polymer/swelling agent compatibility over the temperature range of interest. Temperature-sensitive polymers typically exhibit a lower critical solution temperature (LCST), below which the polymer is soluble. Above this temperature, the polymers are typically hydrophobic and don't swell significantly in water. However, below the LCST, the cross-linked gel swells to significantly higher degrees due to the increased compatibility with water.

Examples: poly (n-isopropyl acryl amide) [27].

\section{Complexing hydrogels}

Some hydrogels may exhibit environmental sensitivity thanks to the formation of polymer complexes. Polymer complexes are insoluble, macromolecular structures formed by the non-covalent association of polymers with an affinity for each other. The complexes form as a result of the association of repeating units on different chains (inter polymer complexes) or separate regions of an equivalent chain (intra polymer complexes). Polymer complexes are classified by the character of the association's stereo complexes, polyelectrolyte complexes, or hydrogen-bonded complexes [28]. The steadiness of the associations depends on such factors because the nature of the swelling agent, temperature, sort of dissolution medium, $\mathrm{pH}$ and ionic strength, network composition and structure, and length of the interacting polymer chains. during this sort of gel, complex formation leads to the formation of physical cross-links within the gel because the degree of effective cross-linking is increased, the network mesh size and degree of swelling is significantly reduced. As a result, if hydrogels are used as drug carriers, the speed of drug release will decrease dramatically abreast of the formation of inter polymer complexes.

Examples: poly (MAA-g-EG) [29]. 


\section{Insitu hydrogels}

Recent advancement in hydrogel engineering has led to the event of in-situ hydrogel formation for drug delivery applications. The in-situ sol-gel transition enables the surgery or implantation procedure to be performed during a minimally invasive manner. Various physical and/or chemical cross-linking mechanisms are used for insitu network formation. Natural phenomenon involved within the formation of in-situ hydrogels are as follows: like Hydrogen bonding, Hydrophobic-hydrophobic interactions, and Electrostatic interactions.

Example, sodium alginate hydrogels are physically formed by crosslinking on the addition of calcium ions but are unstable and disintegrate rapidly and unpredictably [30].

\section{Preparation of hydrogels}

\section{Technologies implemented within the preparation of hydrogels}

On the whole, hydrogels are often formulated from either synthetic polymers or natural polymers. The synthetic polymers are hydrophobic in nature and chemically stronger as compared to natural polymers [31]. Their mechanical strength brings about slow degradation rate, but on the other hand, mechanical strength offers sturdiness also. Through optimum design. These two opposite properties can be balanced. Water-soluble linear polymers of both natural and artificial origin are cross-linked to form hydrogels in various ways like. Linking polymer chains via reaction. Using radiation, Physical interactions like entanglements, electrostatics and crystallite formation.

Mostly the three integral parts of the hydrogels preparation are monomer, initiator and cross-linker. To manage the heat of polymerization and thus the ultimate hydrogels properties, diluents are often employed within the formulation, like water or other aqueous solutions [32]. Hydrogels are normally prepared from polar monomers. according to their starting materials, they're going to be categorized into natural polymer, synthetic polymer and combinations of the two[33].

\section{Bulk polymerization}

Many vinyl monomers can possibly be used for the fabrication of hydrogels. Bulk hydrogels are often obtained with one or more kinds of monomers. Ordinarily, a little amount of cross-linking agent is supplemented for hydrogel formulation. The polymerization reaction is initiated with the help of radiation, ultraviolet or chemical catalysts. the choice of an appropriate initiator relies upon the type of monomers and solvents getting used. The polymerized hydrogel could even be yielded during a good selection of forms counting the films and membranes, rods, particles and emulsions. Bulk polymerization is that the simple technique, which incorporates only monomer and monomer, soluble initiators. The viscosity of the reaction is significantly enhannced with the conversion that generates the heat during polymerization. These problems are often prevented by regulating the reaction. the bulk polymerization of monomers to make a homogeneous hydrogel yields a glassy, transparent polymer matrix which is extremely tough. When placed in water, the glassy matrix swells to become soft and versatile [34].

\section{Solution polymerization/cross-linking}

In the solution copolymerization/cross-linking reaction, the ionic monomers or neutral monomers are blended with the help of multifunctional cross-linking agent. By UV/IR radiation or by a redox initiator system the polymerization is instigated thermally. The prepared hydrogels require washing with water to eliminate the monomers, oligomers, cross-linking agent, the initiator, the soluble and extractable polymer and other impurities. Phase separation takes place and therefore, the heterogeneous hydrogel is formed when the quantity of water during polymerization is sort of the water content in proportion to the equilibrium swelling. Water, ethanol, water-ethanol mixtures and benzyl alcohol are the usual solvents utilized for the solution polymerization of hydrogels [35].

\section{Suspension polymerization or inverse-suspension polymerization}

Dispersion polymerization could also be a worthwhile technique since the products are acquired as powder or microspheres (beads) and thus, grinding isn't needed. Since water-in-oil $(\mathrm{W} / 0)$ process is chosen in preference to the more common oil-in-water $(0 / W)$, the polymerization is denoted as "inverse suspension". during this method, the monomers and initiator are distributed within the hydrocarbon phase as a consistent mixture. The resin particle size and shape is regulated by the viscosity of the monomer solution, agitation speed, rotor design and dispersant type. Several comprehensive discussions on heterophase polymerizations are published previously. The dispersion is thermodynamically unsteady and necessitates both continuous agitation and addition of a coffee hydrophilic-lipophilic-balance (HLB) suspending agent [36].

Usually, hydrogels formulated by the bulk polymerization will have characteristic weak structure. To strengthen the mechanical properties of a hydrogel, it is often attached on the surface which is coated onto sturdy support. This method comprises the generation of free radicals onto a stronger support surface then polymerizing monomers directly onto it, as a results of which a sequence of monomers are covalently bonded to the support. An assortment of polymeric supports are employed for the synthesis of hydrogel by grafting techniques. Ionizing high energy radiation, like gamma rays and electron beams, has been employed as an initiator to formulate the hydrogels of unsaturated compounds [37].

The radicals on the polymer chains are formed by the irradiation of aqueous polymer solution., hydroxyl radicals are formed by the radiolysis of water molecules, which also attack the polymer chains, results in the formation of the macro-radicals. Recombination of the macroradicals on different chains results in the formation of covalent bonds, so ultimately, a cross-linked structure is obtained. Samples of polymers crosslinked by the radiation method are poly (vinyl alcohol), poly (ethylene glycol), and poly (acrylic acid). The foremost advantage of the radiation initiation over the chemical initiation is that the fabrication of relatively pure and initiator-free hydrogels. Hydrogel technical features the purposeful characteristics of an ideal hydrogel material are highest absorption capacity in saline, Preferred rate of absorption (particle size and porosity) relying on the appliance necessity, topmost absorbency under load (AUL), lowermost soluble content and residual monomer, lowest price, utmost robustness and steadiness within the swelling environment also as during the storage, highest biodegradability without formation of toxic species followed by degradation, $\mathrm{pH}$ neutrality after swelling in water. Colorlessness, odorlessness and absolutely nontoxic, photo stability, Re-wetting competency (if required) the hydrogel has got to be able to refund the imbibed solution or to take care of it; enthusiastic to the appliance requisite (e. g., in agricultural or hygienic applications). Practically, it's unachievable that a hydrogel sample would concurrently fulfill all the above described required features. Actually, the synthetic components for acquiring the utmost level of variety of those features will cause inadequacy of the rest [38].

\section{Applications and uses of hydrogels}

Hydrogels are being used in the development of CDDS Controlled drug delivery systems for a long time. The drug-containing hydrogel whenever comes in to contact with aqueous medium the water gets penetrated in to the system and dissolves the drug.

The main phenomena used here is diffusion through which the dissolved drug is diffused out of delivery systems to surrounding aqueous medium.

The various applications of hydrogels [39] are mentioned below fig. 1.

\section{Recent advances}

\section{Wound healing and wound dressing}

Hydrogels have the power to soak up and retain foreign bodies like bacteria in its network structure. Thus, it can act as a moist wound dressing material. In further applications, hydrogels have the tendency to adsorb and eliminate dead tissues which convince be a completely unique feature for defending wounds [40]. Fibroblast 
proliferation is enhanced by reducing fluid loss from the wound surface with the help of hydrogels. Hydrogels lack mechanical strength but full-fill all the standards to be used as an impressive wound dressing material [41]. Permeability, flexibility, and occlusive properties gradually depend upon the swelling characteristics of varied engineered hydrogels the primary hydrogel-based wound dressing material formulated was hydron. another samples of dressings prepared from HEMA monomers together with other polymers like PEG, acrylamide, or agar are Gel perm, Intrasite, Biolex, and Vigilon [42].

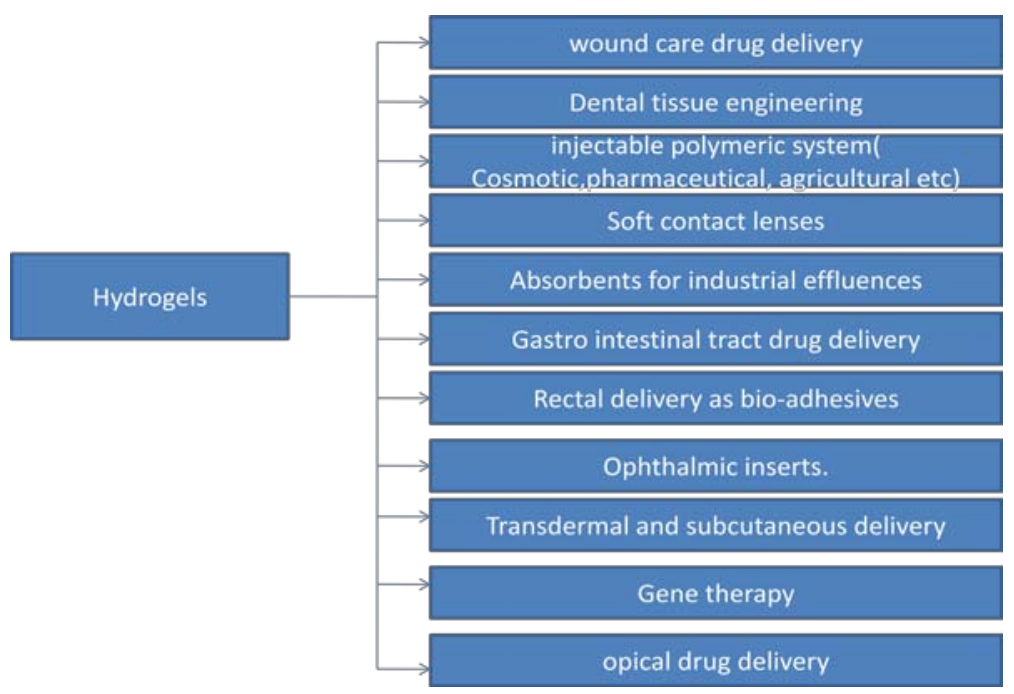

Fig. 1: Applications of hydrogels

\section{Tissue engineering}

Hydrogels likely resemble natural tissues thus emerged together of the abundantly used scaffold biomaterials. Tissue engineering has received increased focus in recent decades due to the utilization of resorbable hydrogels. Thus often since the properties of a hydrogel can be modified effortlessly [43]. Tissue engineering involves both synthetic and natural polymer hydrogels to revive cartilage, tendon, ligament, skin, blood vessels, and heart valves. Naturally derived hydrogels comprise of collagen, silk, keratin, agarose, fibrin, alginate, chitosan, gelatin, and HA, whereas the synthetic hydrogels contains polyurethanes, PEO, PVA, PAAc, and poly (propylene fumarate-coethylene glycol) [44].

\section{Ophthalmology}

Most of the ophthalmic drugs on a mean constitute eye drops or eye ointments. These drugs fail to deliver drugs efficaciously to the intraocular tissue due to certain drawbacks. On the opposite hand, corneal tissue irritation is one among the familiar problems raised by eye ointments. Therefore, exploring alternate drug delivery strategy helps to beat these drawbacks by exploring particles, films, hydrogels, and get in touch with lenses [45].

\section{Immuno-isolation}

Immuno-isolation simply deals with encapsulating a living cell within a semipermeable barrier that allows the bidirectional passage of gases and metabolites but regulating the entry of huge molecules and immunoglobulin[46]. Immuno-isolation mechanically chunks the entry of immunocytes which help to swamp the restrictions of implanting free cells. supported the idea of immune isolation and immune protection, Chang, 1964, was the primary to construct a bioartificial organ using hydrogel. Immuno-isolation of transplanted cells secreting hormones, neurotransmitters, protein, and other bioactive cellular secretary products has raised the advancement of varied gel devices for wider purposes [47].

\section{Cancer therapy}

Hydrogel microparticles have the power to increase and target drugs, which reduce the body's burden remarkable. Since faster and greater swelling is required, superporous hydrogels are going to be more useful than normal hydrogels [48]. For direct administration, like within the case of intratumoral administration, hydrogels are often directly transplanted or injected into the tumor. This increases the efficacy of the anticancer agent. Hydrogel implantation is often used as an alternate approach for multiple dose conventional therapies [49].

\section{CONCLUSION}

Recently, many hydrogel based networks are designed and tailored to satisfy the requirements of various applications. The favorable property of those hydrogels is either ability to swell when put in touch with an solution. The presented review demonstrates the literature concerning classification of hydrogels on different bases, physical and chemical characteristic0s of those products and technical feasibility of the utilization. It also involved technologies adopted for hydrogel production alongside process design implications, block diagrams and optimized conditions of the preparation process. Recent advances of hydrogels was also presented in some details. Super-porous hydrogels are new materials that, no matter their original size, rapidly swell to an outsized size. Different generations of SPHs evolved to deal with the needs surely applications. supported the literature survey, it are often concluded that batch or semi-batch reactors are suitable reactors for polymerization processes. The variables or batch reactors include temperature, pressure, batch cycle time, the quantity of reactants, and therefore the feed addition strategy. Optimization variables like batch cycle time and amount of reactant are continuous variables with fixed values for a particular batch reactor system depends mainly upon material and energy balance. Ribbon mixer with a screw round the axis, screw mixer with four baffles, and double ribbon mixer are three Impellers known to be effective in high viscosity ranges.

\section{FUNDING}

\section{Nil}

\section{AUTHORS CONTRIBUTIONS}

All the authors have contributed equally.

\section{CONFLICT OF INTERESTS}

\section{Declared none}

\section{REFERENCES}

1. S Hoffman. Hydrogel: preparation, characterization and applications. J Adv Res 2015;6:105-21.

2. Ahmed EM. Hydrogel: preparation, characterization and applications. J Adv Res 2015;6:105-21. 
3. Ajji Z, Mirjalili G, Alkhatab A, Dada H. Use of electron beam for the production of hydrogel dressings. Radiat Phys Chem 2008;77:200-12.

4. Bennett SL, Melanson DA, Torchiana DF, Wiseman DM, Sawhney AS. Next-generation hydrogel films as tissue sealants and adhesion barriers. J Cardiac Surgery 2003;18:494-9.

5. Byju AG, Kulkarni A, Gundiah N. Mechanics of gelatin and elastin based hydrogels as tissue engineered constructs. Int Conference Fracture 2013;18:524-50.

6. Das N. Preparation methods and properties of hydrogels: a review. Int J Pharm Pharm Sci 2013;5:112-7.

7. Hydrogel: Properties, preparation and technical features. Asian J Biomaterial Res 2016;2:163-70.

8. Okay, general properties of hydrogels. In: Gerlach G, Arndt KF. Hydrogel sensors and actuators, engineering and technology. Springer Series Chem Sensors Biosensors 2010;3:173-80.

9. Byju AG, Kulkarni A, Gundiah N. Mechanics of gelatin and elastin based hydrogels as tissue engineered constructs. Materials Science 2013;5:250-94.

10. Mondal M, Trivedy K, Nirmal Kumar S. The silk proteins, serin and fibroin in silkworm Bombyxmori. Caspian J Environ Sci 2007;5:63-76

11. Menard KP. Dynamic mechanical analysis: a practical introduction. $2^{\text {nd }}$ ed. CRC Press; 2008. p. 480-540.

12. Morrison FA. Understandin rheology. Oxford University Press; 2013:5:250-94.

13. Dong LC, Hoffman AS, Yan Q. Dextran permeation through poly (N-isopropylacrylamide) hydrogels. J Biomater Polym; 1994. p. 473-84.

14. ASTM International. F2540. Standard practice standard guide for assessing microstructure of polymeric scaffolds for use in tissue engineered medical products; 2004. p. 600-29.

15. ASTM International. F2603 standard practice Standard Guide for Interpreting Images of Polymeric Tissue Scaffolds; 2006. p. 120-80.

16. Gulrez SKH, Al-Assaf S, Phillips GO. Hydrogels: methods of preparation, characterisation and applications, progress in molecular and environmental bioengineering. In: Carpi A. Analysis and modeling to technology applications; 2011. p. 65-94.

17. Hoffman AS. History, applications and uses of hydrogels. Adv Drug Delivery Rev 2001;3:3-12.

18. Bian L, Hou C, Tous E, Rai R, Mauck RL. The influence of hyaluronic acid hydrogel crosslinking density and macromolecular diffusivity on human MSC chondrogenesis and hypertrophy. Biomaterials 2013;34:413-21.

19. Sung HW, Huang DM, Chang WH, Huang RN, Hsu JC. Evaluation of gelatin hydrogel crosslinked with various crosslinking agents as bioadhesives: in vitro study. J Biomed Mater 1999:46:520-30.

20. Weber LM, Lopez CG, Anseth KS. Effects of PEG hydrogel crosslinking density on protein diffusion and encapsulated islet survival and function. J Biomed Mater 2009;90:720-9.

21. Naziha Chirani, L'Hocine Yahia, Lukas Gritsch, Federico Leonardo Motta, Soumia Chirani, Silvia Fare. History and applications of hydrogels. J Biomed Sci 2015;N2:13.

22. Syed KH Gulrez, Saphwan Al-Assaf, Glyn O Phillips. Hydrogels: methods of preparation, characterisation and applications. Prog Mol Environ Bioeng: Anal Model Technol Appl 2011;5:655-60.

23. Zhou Y, Chu JS, Wu XY. Theoretical analysis of drug release into a finite medium from sphere ensembles with various size and concentration distributions. Eur J Pharm 2004;22:251-9.

24. Katchalsky, I Michaeli. Polyelectrolyte gels in salt solution. J Polym 1955;15:69-86.

25. L Brannon Peppas, NA Peppas. Equilibrium swelling behaviour of pH-sensitive hydrogels. Chem Eng 1991;46:715-22.
26. Katchalsky. Rapid swelling and deswelling of reversible gels of polymeric acids by ionization. Experimentia 1949;5:319-20.

27. Katchalsky S Lifson, H Eisenberg. Equation of swelling for poly electrolyte gels. J Polym 1951;7:571-4.

28. T Tanaka. Phase transition in gels and a single polymer. Polymer 1979;20:1404-12.

29. L Brannon Peppas, NA Peppas. The equilibrium swelling behaviour of porous and non-porous hydrogels. In: L Brannon Peppas, RS Harland. Eds. Absorbent Polymer Technology, Elsevier, Amsterdam; 1990. p. 67-75.

30. W Oppermann. Swelling behaviour and elastic properties of ionic hydrogels. In: RS Harland, RK Prud' home. Eds. Polyelectrolyte Gels: Properties, Preparation, and Applications, ACS Symposium Series, No. 480, American Chemical Society, Washington, DC; 1992. p. 159-70.

31. Nagam SP, AN Jyothi, J Poojitha, S Aruna, RR Nadendla. A comprehensive review on hydrogels. Int J Curr Pharm Res 2016;8:19-23.

32. Ahmed EM. Hydrogel: preparation, characterization and applications. J Adv Res 2015;6:105-21.

33. Dong LC, Hoffman AS, Yan Q. Dextran permeation through poly (N-isopropylacrylamide) hydrogels. J Biomaterials Sci 1994;5:473-84.

34. Griffith LG. Polymeric biomaterials. Recent advances in hydrogels for biomedical applications. Asian J Pharm Clin Res Acta Materialia 2000;48:263-77.

35. Hoffman AS. Hydrogels for biomedical applications. Adv Drug Delivery Rev 2012;64:18-23.

36. Hacker MC, Mikos AG. Synthetic polymers, principles of regenerative medicine. $2^{\text {nd }}$ ed.; 2011. p. 587-622.

37. Gulrez SKH, Al-Assaf S, Phillips GO. Hydrogels: methods of preparation, characterisation and applications, progress in molecular and environmental bioengineering. In: Carpi A. Analysis and modeling to technology applications; 2011. p. 74-96.

38. Sweta Garg, Ashish Garg. Hydrogel: classification, properties, preparation and technical features. Asian J Biomaterial Res 2016;2:163-70.

39. Nagam. Hydrogel: classification, properties, preparation and technical features. Int J Curr Pharm 2016;1:19-23.

40. Silva AK, Richard C, Bessodes M, Scherman D, Merten OW. Growth factor delivery approaches in hydrogels. Biomacromolecules 2009;10:918.

41. Ratnaparkhi PK, Prajapati VK, Jani GK, Solanki HK. Recent expansions in an emergent novel drug delivery technology: hydrogel. World J Pharm Pharm Sci 2015;4:678-701.

42. Kishida A, Ikada Y. Hydrogels for biomedical and pharmaceutical applications. In: Dumitriu S. editor. Polymeric Biomaterials. 2nd ed. Ch. 6. New York: Marcel Dekker; 2002. p. 504-20.

43. Brown AS. Hydron for burns. Hydrogels for tissue engineering. Plast Reconstruct Surg 1981;67:810-1.

44. Yates DW, Hadfield JM. Clinical experience with a new hydrogel wound dressing. Injury 1984;16:23-4.

45. Myers JA. Delperm: a nontextile wound dressing. Pharm J 1983;230:263.

46. Lee KY, Mooney DJ. Hydrogels for tissue engineering. Chem 2001;101:1869-79.

47. Kumaran P, Gupta A, Sharma S. Synthesis of wound-healing keratin hydrogels using chicken feathers proteins and its properties. Int J Pharm Pharm 2017;9:171-8.

48. Azevedo EP. Chitosan hydrogels for drug delivery and tissue engineering applications. Int J Pharm Pharm Sci 2015;7:8-14.

49. Lim F, Sun AM. Microencapsulated islets as bioartificial endocrine pancreas. Science 1980;210:908-10.

50. Roy Debansh. Recent advances in hydrogels for biomedical applications. Asian J Pharm Clin Res 2018;11:410-34. 